\title{
Surface Nanobubbles Nucleate Microdroplets
}

\author{
Xuehua Zhang, ${ }^{1,2, *}$ Henri Lhuissier, ${ }^{2}$ Chao Sun, ${ }^{2}$ and Detlef Lohse ${ }^{2, \dagger}$ \\ ${ }^{1}$ Department of Chemical and Biomolecular Engineering, University of Melbourne, Parkville, Victoria 3010, Australia \\ ${ }^{2}$ Physics of Fluids Group, Department of Applied Physics, and J. M. Burgers Centre for Fluid Dynamics, University of Twente, \\ P.O. Box 217, 7500 AE Enschede, The Netherlands
}

(Received 30 September 2013; published 10 April 2014)

\begin{abstract}
When a hydrophobic solid is in contact with water, surface nanobubbles often form at the interface. They have a lifetime many orders of magnitude longer than expected. Here, we show that they even withstand a temperature increase to temperatures close to the boiling point of bulk water; i.e., they do not nucleate larger bubbles ("superstability"). On the contrary, when the vapor-liquid contact line passes a nanobubble, a liquid film remains around it, which, after pinch-off, results in a microdroplet in which the nanobubbles continue to exist. Finally, the microdroplet evaporates and the nanobubble consequently bursts. Our results support that pinning plays a crucial role for nanobubble stability.
\end{abstract}

DOI: 10.1103/PhysRevLett.112.144503

PACS numbers: 47.55.dp

Surface nanobubbles [1-6] are nano- to microscale, gaseous domains adsorbed on a solid surface in contact with water. The intense research interest in surface nanobubbles arises from their potential applications in microfluidics [7-9] and the scientific challenge for understanding their fundamental physical properties. One of the most pronounced features of surface nanobubbles is their very long lifetime - up to days, rather than the microseconds one expects given their size. Different mechanisms have been proposed to rationalize the stability of nanobubbles [1014]. For example, the stability of nanobubbles was attributed to the presence of the impermeable skin on the bubble surface [11]. However, from the systematic measurements after rinsing the system with various detergents, no evidence was found that nanobubbles were stabilized by the insoluble organic contaminants $[15,16]$. In the dynamic equilibrium hypothesis, the gas outflux from the bubbles is suggested to be balanced by a gas influx. However, recent particle tracking measurements-although with limited spatial resolution-do not give any indication for an induced liquid flux around the bubble [17,18]. Recent findings have suggested that the pinning of the three-phase nanobubble-liquid-surface contact line and the collective effect from neighboring nanobubbles can be very important in the long lifetimes of nanobubbles [12-14].

It is unknown how surface nanobubbles respond to a large temperature increase and whether they can influence some interfacial events during boiling, such as surface drying from evaporation and droplet condensation. In this Letter, we show that surface nanobubbles withstand an increase of temperature up to the boiling point of bulk water. Even more remarkably, when a macroscopic surface bubble passes a nanobubble during the boiling process, a liquid film remains around it, which, after pinch-off, results in a microdroplet in which the nanobubbles continue to exist. Such microdroplets inside the macroscopic surface bubble further grow by condensation. Our results demonstrate the stability of nanobubbles at close-to-boiling temperatures and their persistence even after the bulk water has receded from the surface due to the shielding liquid microdroplet, again suggesting that pinning plays a crucial role in nanobubble stability, both with respect to their position and their lifetime. Our results even reveal that nanobubbles can stably exist in micron-sized droplets, which is to our knowledge the smallest volume in which they have hitherto been observed. An artist's impression of the whole process is given in the Supplemental Material [19].

Experimental procedure and observations.-We created surface nanobubbles by the standard solvent exchange process [20-23]. A diagram of the experimental setup is shown in Fig. 1. Nanobubbles were produced in airsaturated water at $37^{\circ} \mathrm{C}$ either on the hydrophobic glass window above the liquid or on a second hydrophobic substrate below it. The size distribution of nanobubbles was first characterized by atomic force microscopy (AFM) and

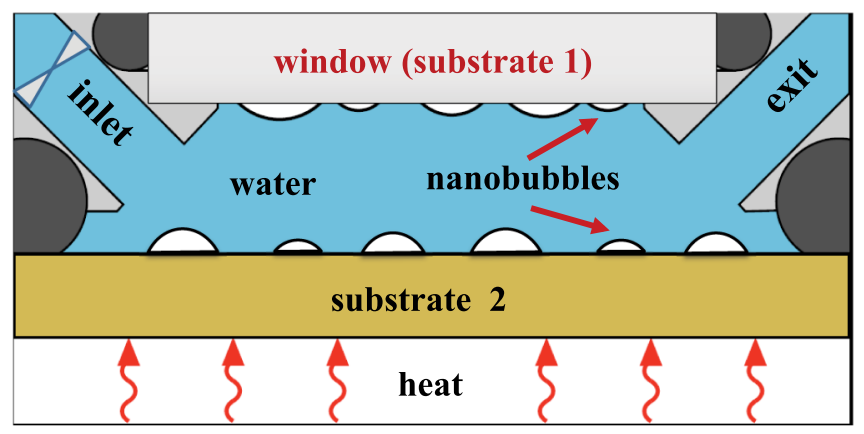

FIG. 1 (color online). Experimental setup. The nanobubbles were produced on substrate 1 [octadecyltrimethylchlorosilane (OTS) glass] for the bottom view on the bubbles or on substrate 2 (OTS silicon) for the top view on them. Imaging with optical microscopy and a high-speed camera is always from above. 
is provided in the Supplemental Material [19]. In addition, with optical microscopy, we can resolve those nanobubbles with lateral radius $R$ larger than $0.75 \mu \mathrm{m}$ (radius of the circular contact area between the nanobubble and the substrate). The liquid with the nanobubbles was then heated. Within the optical resolution limits, we could not observe any noticeable change (growth or shrinkage) of the nanobubbles with an increase in temperature from $37^{\circ} \mathrm{C}$ to $74^{\circ} \mathrm{C}$, neither from the bottom view on the bubbles nor from the top view on them. At $74^{\circ} \mathrm{C}$, the size, number density, and location of the nanobubbles remained unchanged for $10 \mathrm{~h}$. The in situ AFM imaging allowed us to follow the nanobubbles up to $80^{\circ} \mathrm{C}$, where they still stayed on the surface. For more details on the experimental procedure, please refer to the Supplemental Material [19].

By optical imaging, we found that the nanobubbles actually remained stable until macroscopic surface bubbles form on the substrate at $90{ }^{\circ} \mathrm{C}$. They must contain both vapor (at a partial pressure just as above the liquid or in the nanobubbles) and air, as the water at these temperatures is oversaturated with dissolved air. A typical macroscopic surface bubble grew and moved across the surface, separating wet regions (outside the bubble) from dry regions (inside the bubble). When it moved towards a region where surface nanobubbles had been observed in the wet phase, at every single nanobubble position, a microdroplet was observed in the dry phase. Figure 2(a) shows the nanobubbles in water and Fig. 2(b) the corresponding microdroplets in the gas phase at the very same positions of the nanobubbles, which persist inside the microdroplets (and, in addition, microdroplets nucleated by normal condensation). Apparently, the surface nanobubble had triggered the nucleation of a microdroplet in the macroscopic bubble, in which the droplets further condense. The latter is similar to the situation as described by Zhong et al. [24]. The microdroplet nucleation happens basically for every single surface nanobubble. (A full statistical analysis is given in the Supplemental Material [19].)

How does the dynamics of this droplet nucleation process work? In Figs. 2(c)-2(f), we show snapshots of the three-phase contact line (TPCL), which separates the macroscopic bubble from the bulk liquid, as it moves across the nanobubbles - the whole dynamical process can best be seen in a Supplemental Movie (M1) [19]: The TPCL initially recedes at a rate of $1.5 \mu \mathrm{m} / \mathrm{s}$ and is then pinned when it reaches a nanobubble, as clearly indicated by the distorted fringes in Fig. 2(d). The TPCL then snaps off from the nanobubble and recedes further, clearly demonstrating that the nanobubbles are strongly pinned to the surface. The snap-off of the TPCL is followed by the immediate nucleation of a water droplet exactly at the location of the nanobubble. In Fig. 2(f), the water droplet has grown to a lateral radius of $2 \mu \mathrm{m}$ through further vapor condensation, showing that the gas inside the expanding macroscopic bubble is supersaturated with vapor.
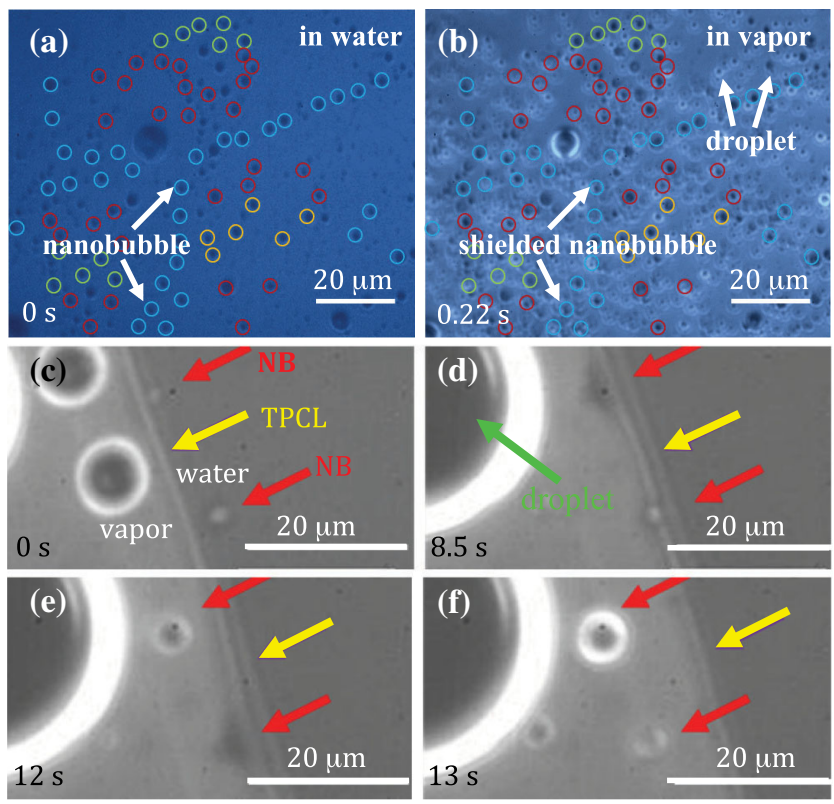

FIG. 2 (color online). (a) Optical image of nanobubbles in the water phase at $90^{\circ} \mathrm{C}$ and (b) the corresponding image of the very same location after the TPCL has passed with microdroplets in the gas phase, with the entrapped nanobubbles. In (b), the temperature is $95^{\circ} \mathrm{C}$. The nanobubbles and resulting droplets are marked with various colors to assist recognition. The patterns of the marks on the surface in (a) are identical to those in (b), demonstrating that all nanobubbles nucleate microdroplets. Also shown are some spontaneously nucleated microdroplets. (c)-(f) Optical bottom view snapshots of the dynamical process of microdroplet nucleation through the nanobubbles and of the subsequent growth of the microdroplets at $90{ }^{\circ} \mathrm{C}$. The TPCL (yellow arrow) moves from the left to the right across the nanobubbles [NB, marked with red arrows (darker arrows in the printed version) in (c) and (d)], leading to microdroplets [red arrows in (e) and, after further growth by condensation, in (f)] with the entrapped surface nanobubbles.

The bottom view on the bubbles of Fig. 2 has provided clear images of the boundary between the liquid phase and the gas phase, of the pinning, and of the snap-off of the three-phase line. The pinning of the TPCL by the (evidently pinned) nanobubbles and its role in the nucleation of droplets on nanobubbles are further investigated from the top view on the bubbles; i.e., the substrate was placed on the bottom of the fluid cell and it was completely dry by the end of boiling. Figures 3(a)-3(d) show the initial stage of boiling as the substrate is alternatingly dried by vaporization and wetted by condensation. A nanobubble distorts the receding TPCL until a "peninsula" of water is left behind, which subsequently pinches at its narrowest part to form an isolated droplet containing the nanobubble. With time, the amount of liquid around the nanobubbles increases, showing the condensation under the highly humid conditions inside the fluid cell. The second row of Fig. 3 gives a schematics of the whole process. 
(a)

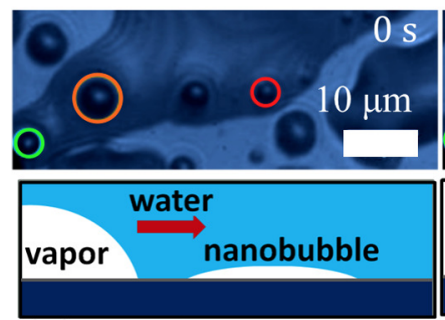

(e) (b)

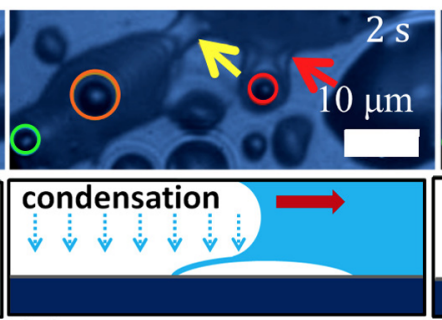

(f) (c)

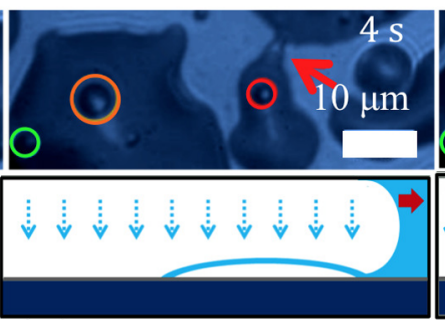

(g) (d)

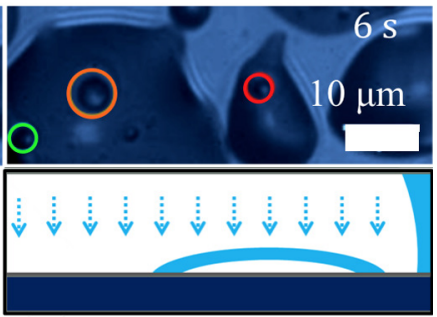

(h)

FIG. 3 (color online). Top view snapshots of nanobubbles in water at $95^{\circ} \mathrm{C}$. Individual nanobubbles are identified by different colors, and the arrows point to the liquid bridges which eventually break. The corresponding times are (a) $0 \mathrm{~s}$, (b) $2 \mathrm{~s}$, (c) $4 \mathrm{~s}$, and (d) $6 \mathrm{~s}$. (e)-(h) Schematic drawings of the microdroplet nucleation by nanobubbles at high humidity.

Up to now, we have described how a nanobubble nucleates a microdroplet inside the vapor-oversaturated phase. We now address the eventual fate of the nanobubbles inside the microdroplets (which are inside the macroscopic bubble) as the boiling proceeds. Figures 4(a)-4(d) show the series of events in the final stage of boiling with the temperature increasing from $97^{\circ} \mathrm{C}-100{ }^{\circ} \mathrm{C}$, immediately before the water dried out, i.e., when the relative humidity in the environment became so low that the droplet evaporation prevailed over the vapor condensation. In Fig. 4(a), the receding TPCL is pinned by a nanobubble and then pinches off, as before. But, under the conditions of low humidity, the nanobubble then bursts and leaves a puddle of water on the surface [Fig. 4(b)], as similarly observed for the bursting of macroscopic bubbles $[25,26]$. The puddle finally dries with time. A similar motion of the TPCL across a nanobubble and the depinning-bursting sequence is shown in Figs. 4(c) and 4(d). A movie of this process is provided in the Supplemental Material (M3) [19].

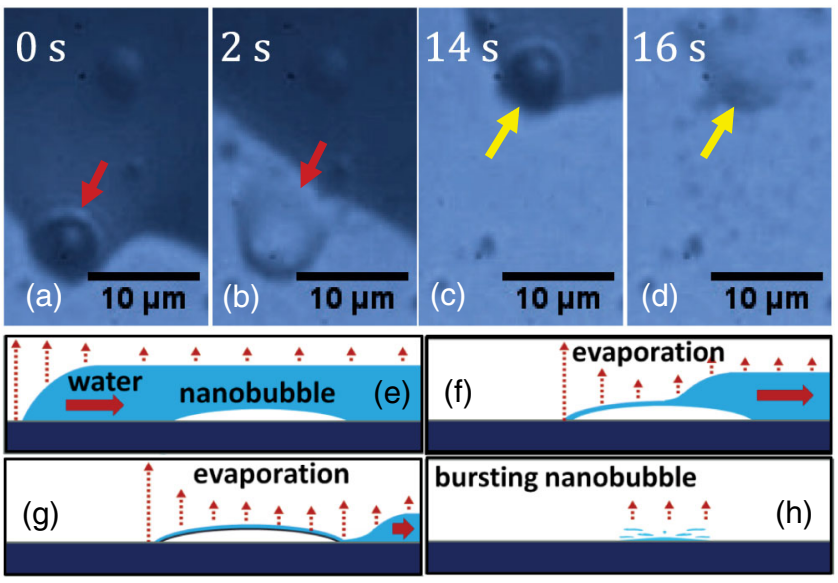

FIG. 4 (color online). Bursting of nanobubbles at $97^{\circ} \mathrm{C}-100^{\circ} \mathrm{C}$. (a)-(d) Top view snapshots of nanobubbles through which the TPCL passes: It first remains pinned; then, a microdroplet with an entrapped nanobubble emerges, which finally bursts. (e)-(h) Schematic drawings of bursting nanobubbles, which occurs under low humidity conditions.
Interpretation and theoretical considerations.-(i) Superstability of surface nanobubbles under boiling conditions: Previous work has shown that nanobubbles live for days under ambient conditions and are even stable under a massive reduction of the liquid pressure down to $-6 \mathrm{MPa}$ [27,28]. The bubble nucleation theory of Borkent et al. [29] shows that nanobubbles in artificial nanopits nucleate at pressures exactly as theoretically calculated [Fig. 5(a)], in contrast to surface nanobubbles which sustain even much larger pressures [27]. This latter observation has been called superstability. Here, rather than by reducing the pressure, we try to induce the nucleation of a vapor bubble from a nanobubble by means of a temperature increase. We now calculate, for a given temperature increase, the bubble size threshold beyond which surface tension should be too weak to prevent the explosive growth of the surface nanobubble due to vapor formation: At the initial temperature $T_{i}$, the ambient pressure $p_{\text {amb }}$ and surface tension balance the initial gas pressure $p_{g}\left(T_{i}\right)$ and the initial vapor pressure $p_{v}\left(T_{i}\right)$. At the final temperature $T_{f}$, the latter two
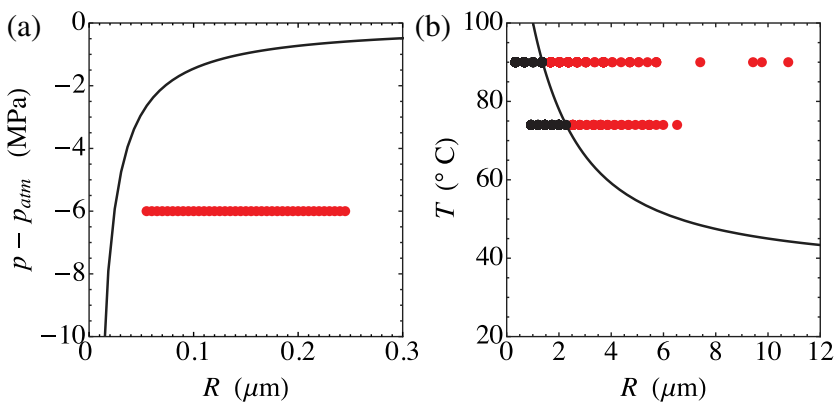

FIG. 5 (color online). Superstability of surface nanobubbles (a) under pressure reduction and (b) under temperature increase. In Ref. [29], the pressure below which the nucleation is expected was calculated [solid line in (a)]. However, the surface nanobubbles (data taken from Ref. [27]) were stable [red data points in (a)]. Here, the temperature calculated from Eq. (1) is supposed to trigger vapor bubble formation beyond the solid line in (b). However, most of the nanobubbles are stable (red data points, taken from our movies). The black data points represent bubbles which are stable, in accordance with the expectation. 
increase to $p_{g}\left(T_{f}\right)$ and $p_{v}\left(T_{f}\right)$, respectively. To avoid unlimited growth, the pressure increase $\Delta p\left(T_{i} \rightarrow T_{f}\right)$ must be balanced by the Laplace pressure $2 \sigma\left(T_{f}\right) / R$, leading to

$$
R_{c}\left(T_{f}\right)=2 \sigma\left(T_{f}\right) / \Delta p\left(T_{i} \rightarrow T_{f}\right),
$$

as the critical lateral radius beyond which all bubbles should grow at a given $T_{f}$ [30]. We calculated $R_{c}\left(T_{f}\right)$ according to Eq. (1) in Fig. 5(b) and compare the result with the size of the bubbles we detected in the same figure: We find that many of the bubbles detected in the experiments are in the unstable regime but nonetheless do not grow; i.e., they are superstable. We have thus established that nanobubbles are superstable with respect not only to pressure reduction but also to temperature increase. Clearly, further stabilizing forces such as pinning (presumably due to intrinsic geometrical or/and chemical heterogeneities of the surface) must play a role, leading to (i) the positional stability of the nanobubbles, (ii) their long lifetimes [13], and (iii) the observed strong contact line hysteresis of $20{ }^{\circ} \mathrm{C}-30{ }^{\circ} \mathrm{C}$ between the (macroscopic) advancing and receding contact angles (see the Supplemental Material [19] for details).

(ii) Nanobubble induced droplet formation: Next, we discuss the possible mechanism of the nanobubble-initiated droplet nucleation. The proposed mechanism is depicted in Figs. 3(e)-3(h): As boiling leads to the formation of macroscopic vapor bubbles, the boundary of such an expanding bubble moves across a nanobubble [Figs. 3(e) and 3(f)]. However, due to the strong pinning to the substrate, the nanobubbles survive the crossing of the TPCL. During the collision between a macroscopic bubble and a nanobubble, a thin water film can indeed remain on the surface of the nanobubble due to the slow hydrodynamic drainage [31]. The stability of such thin films has been studied for head-on collisions between two bubbles [32-34], often in the context of process technology [35], but even then the phenomenon is not fully understood [36]. Our situation here represents a much more complex geometry in which a nanobubble pinned on the substrate encounters a vapor-dominated phase with a nearly straight TPCL (i.e., a large vapor bubble). We predict that the stability of nanobubbles depends on the collision rate: at a very slow rate, the hydrodynamic drainage occurs and nanobubbles coalesce with the vapor phase. A quantitative measurement is yet to be designed for a better understanding of this phenomenon.

In the highly humid atmosphere inside the vapor bubble, nanobubbles shielded with the thin liquid film act as nuclei for further vapor condensation, which results in microdroplets growing inside the vapor bubble [Figs. 3(g) and 3(h)]. At the same time, the further expansion of the macroscopic bubble stretches the liquid bridge connected to the nanobubble and finally leads to the pinch-off of the receding TPCL and the isolation of a droplet containing the nanobubble [Fig. 3(h)]. The persistence of the nanobubbles is a consequence of their protective shielding by a water film. This again suggests that nanobubbles are strongly pinned on the substrate. The proposed shielding film mechanism also explains why the nucleation of droplets by nanobubbles is observed in the early stages of boiling on both the bottom substrate and the top glass window. In both cases, the rapid condensation at geometric irregularities provided by the nanobubbles occurred due to the high humidity within the vapor bubble, as evidenced by the growth of the droplets.

(iii) Nanobubble bursting: Figures 4(e)-4(h) illustrate a possible process for nanobubble bursting. Although nanobubbles still retain a thin layer of water immediately following the retraction of the TPCL, this thin film is not stable. In the absence of vapor condensation onto the nanobubbles behind the TPCL due to a less humid environment, evaporation leads to the thinning and eventual rupture of the liquid film and bursting of the nanobubbles. Therefore, whether nanobubbles nucleate droplets or pop depends on the relative humidity in the large vapor bubble which changes over boiling time. We note that the bursting phenomenon is additional evidence for the gaseous nature of nanobubbles, as nanodroplets could not burst.

Summary and outlook.-To summarize, nanobubbles are stable with respect to a temperature increase close to the boiling point of water and pin the drying front of water receding over the surface, as they are themselves pinned. Remarkably, they can trigger microdroplet nucleation at high humidity in the evaporation process. We have thus demonstrated for the first time the unique role of nanoscale gaseous domains in boiling events. This work is a starting point for studying the impact of nanoscale fluidic domains on the motion of the receding three-phase line and for understanding the phase transition initiated by surface nanobubbles.

We gratefully acknowledge Andrea Prosperetti for inspiring discussions. X.Z. acknowledges support from the Australian Research Council and D. L. from a European Research Council (ERC) Advanced Grant. We thank Chenglong $\mathrm{Xu}$ for the help on XPS and AFM measurements.

*xuehuaz@unimelb.edu.au

†.lohse@utwente.nl

[1] P. Ball, Nature (London) 423, 25 (2003).

[2] P. Attard, Adv. Colloid Interface Sci. 104, 75 (2003).

[3] H. K. Christenson and P. M. Claesson, Adv. Colloid Interface Sci. 91, 391 (2001).

[4] V. S. J. Craig, Soft Matter 7, 40 (2011).

[5] J. R. T. Seddon and D. Lohse, J. Phys. Condens. Matter 23, 133001 (2011).

[6] M. A. Hampton and A. V. Nguyen, Adv. Colloid Interface Sci. 154, 30 (2010). 
[7] P. G. de Gennes, Langmuir 18, 3413 (2002).

[8] V. Belova, D. A. Gorin, D. G. Shchukin, and H. Möhwald, Angew. Chem., Int. Ed. Engl. 49, 7129 (2010); V. Belova, M. Krasowska, D. Wang, J. Ralston, D. G. Shchukin, and H. Moehwald, Chem. Sci. 4, 248 (2013).

[9] I. U. Vakarelski, N. A. Patankar, J. O. Marston, D. Y. C. Chan, and S. T. Thoroddsen, Nature (London) 489, 274 (2012).

[10] M. P. Brenner and D. Lohse, Phys. Rev. Lett. 101, 214505 (2008).

[11] W. A. Ducker, Langmuir 25, 8907 (2009).

[12] X. Zhang, D. Y. C. Chan, D. Wang, and N. Maeda, Langmuir 29, 1017 (2013).

[13] J. H. Weijs and D. Lohse, Phys. Rev. Lett. 110, 054501 (2013).

[14] Y. Liu and X. Zhang, J. Chem. Phys. 138, 014706 (2013).

[15] X. H. Zhang, M. H. Uddin, H. J. Yang, G. Toikka, W. Ducker, and N. Maeda, Langmuir 28, 10471 (2012).

[16] Further evidence against the contamination hypothesis is given in the Supplemental Material [19], Sec. 2. In that section, we also give evidence for the stability of our substrate OTS Si at high temperatures.

[17] C. U. Chan and C.-D. Ohl, Phys. Rev. Lett. 109, 174501 (2012).

[18] E. Dietrich, H. J. W. Zandvliet, D. Lohse, and J. R. T. Seddon, J. Phys. Condens. Matter 25, 184009 (2013).

[19] See Supplemental Material at http://link.aps.org/ supplemental/10.1103/PhysRevLett.112.144503 for an artist's impression of the whole process, materials and methods, bubble size distribution, images at different temperatures, contamination control, and supporting movies.

[20] S.-T. Lou, Z.-Q. Ouyang, Y. Zhang, X.-J. Li, J. Hu, M.-Q. Li, and F.-J. Yang, J. Vac. Sci. Technol. B 18, 2573 (2000).

[21] X. H. Zhang, X. D. Zhang, S. T. Lou, Z. X. Zhang, J. L. Sun, and J. Hu, Langmuir 20, 3813 (2004).
[22] X. H. Zhang, N. Maeda, and V. S. J. Craig, Langmuir 22, 5025 (2006).

[23] X. H. Zhang, A. Khan, and W. A. Ducker, Phys. Rev. Lett. 98, 136101 (2007).

[24] J.-Q. Zhong, D. Funfschilling, and G. Ahlers, Phys. Rev. Lett. 102, 124501 (2009).

[25] H. Lhuissier and E. Villermaux, Phys. Fluids 21, 091111 (2009).

[26] H. Lhuissier and E. Villermaux, J. Fluid Mech. 696, 5 (2012).

[27] B. M. Borkent, S. M. Dammer, H. Schönherr, G. J. Vancso, and D. Lohse, Phys. Rev. Lett. 98, 204502 (2007).

[28] X. H. Zhang, A. Quinn, and W. A. Ducker, Langmuir 24, 4756 (2008).

[29] B. M. Borkent, S. Gekle, A. Prosperetti, and D. Lohse, Phys. Fluids 21, 102003 (2009).

[30] For simplicity and clarity, we have assumed a hemispherical bubble with a contact angle $\theta=90^{\circ}$. Any other angle would yield a smaller critical value $R_{c}\left(T_{f}\right) \sin \theta$ for the lateral radius, which is the quantity we measure, i.e., results in even more bubbles being considered superstable.

[31] V. V. Yaminsky, S. Ohnishi, E. A. Vogler, and R. G. Horn, Langmuir 26, 8061 (2010); I. U. Vakarelski, R. Manica, X. Tang, S. J. O'Shea, G. W. Stevens, F. Grieser, R. R. Dagastine, and D. Y. C. Chan, Proc. Natl. Acad. Sci. U.S.A. 107, 11177 (2010).

[32] L. Doubliez, Int. J. Multiphase Flow 17, 783 (1991).

[33] N. Bremond, M. Arora, S. M. Dammer, and D. Lohse, Phys. Fluids 18, 121505 (2006).

[34] A. Kamp, A. Chesters, C. Colin, and J. Fabre, Int. J. Multiphase Flow 27, 1363 (2001).

[35] D. Y. C. Chan, E. Klaseboer, and R. Manica, Soft Matter 7, 2235 (2011).

[36] V.S. Craig, Curr. Opin. Colloid Interface Sci. 9, 178 (2004). 